\title{
Targeted Alpha Particle Therapy for Neuroendocrine Tumours: The Next Generation of Peptide Receptor Radionuclide Therapy
}

\author{
Shaunak Navalkissoor ${ }^{\mathrm{a}}$ Ashley Grossman ${ }^{\mathrm{b}}$ \\ a Department of Nuclear Medicine, ENETS Centre of Excellence, Royal Free Hospital, London, UK; \\ ${ }^{b}$ NET Unit, ENETS Centre of Excellence, Royal Free Hospital, London, UK
}

\section{Keywords}

Neuroendocrine tumours . Peptide receptor radionuclide therapy · Alpha particles · Therapy

\begin{abstract}
Neuroendocrine tumours (NETs) are being seen increasingly frequently, but to date only complete surgical resection is curative. However, among the various therapeutic options, peptide receptor radionuclide therapy, linking a radioactive moiety to an octreotide derivative, has been shown to be highly efficacious and a well-tolerated therapy, improving progression-free survival and probably overall survival. Nevertheless, the current radionuclides in use are beta particle emitters with non-optimal radiobiological properties. A new generation of alpha particle-emitting radionuclides is being developed, with advantages in terms of very high energy and a short path length, which should theoretically show higher efficacy. We survey the current developments in this field, emphasising the exciting potential of this novel form of therapy for NETs.

๑2018S. Karger AG, Basel
\end{abstract}

\section{Introduction}

Neuroendocrine tumours (NETs) are heterogeneous tumours with varying clinical behaviour. Therapeutic decisions are generally based on histological and imaging assessments $[1,2]$, together with clinical features. In patients with localised well-differentiated NETs, surgery is a curative option. There is usually no curative surgical option in patients with non-localised metastatic disease. There are several licensed and unlicensed treatment strategies for metastatic NETs that have been shown to provide symptomatic benefit and, in some cases, to prolong progression-free survival and occasionally overall survival. Systemic treatment options include long-acting somatostatin analogues, chemotherapy, molecular targeted treatments (e.g., everolimus and sunitinib), alpha-interferon, and peptide receptor radionuclide therapy (PRRT).

Of these systemic treatments, PRRT has proven to be one of the more promising options, acting as it does to cause targeted DNA damage (see below). PRRT has been available since the early 1990s: the first PRRT radiopeptide employed was to utilise high activity of ${ }^{111}$ In-DTPAoctreotide (Octreoscan ${ }^{\circledR}$ ), a radioisotope more generally used for imaging due to its release of gamma radiation. However, the decay of this radiopeptide results in the re-

\section{KARGER}

(C2018 S. Karger AG, Basel

E-Mail karger@karger.com

www.karger.com/nen
Dr. Shaunak Navalkissoor

Department of Nuclear Medicine

ENETS Centre of Excellence, Royal Free Hospital

London NW3 2QG (UK)

E-Mail s.navalkissoor@nhs.net 
Fig. 1. Schematic diagram of the types of radionuclide decay and consequent different types of radiation used clinically.

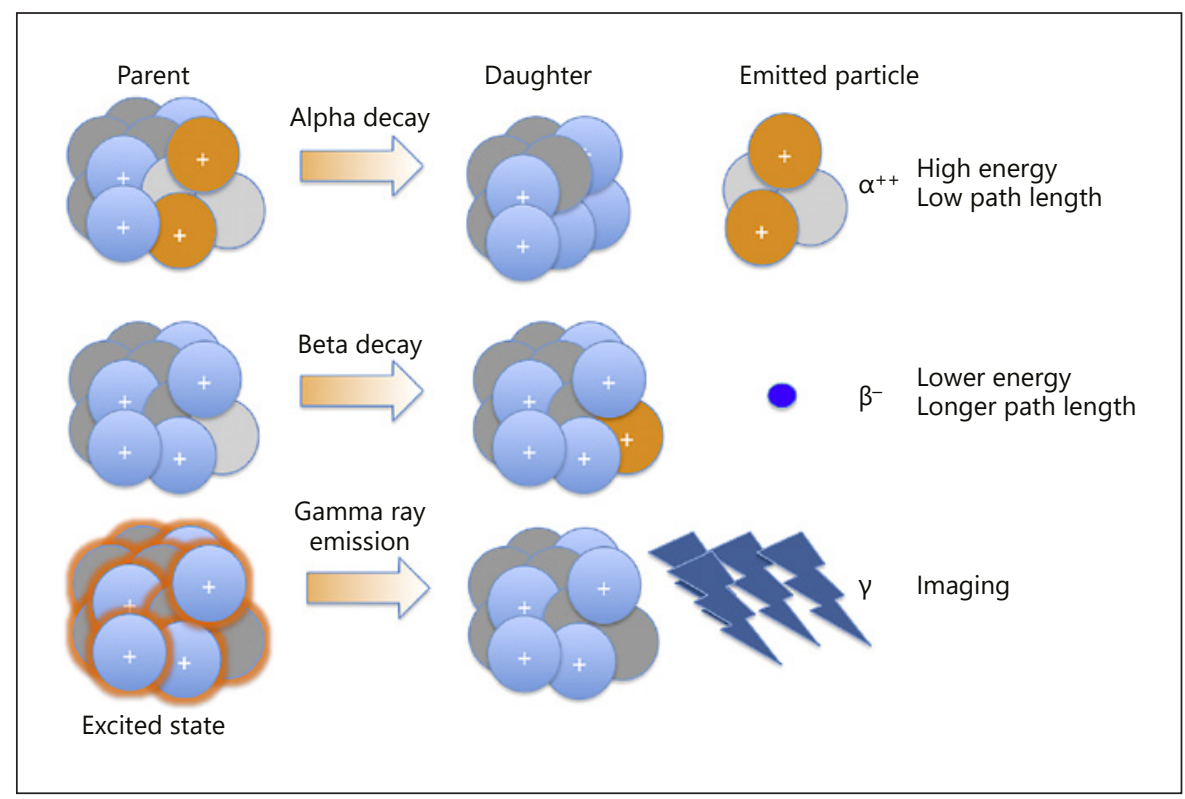

lease of potentially therapeutic short-range Auger electrons, leading to localised cellular damage. Although treatment with ${ }^{111}$ In-DTPA-octreotide resulted in reasonable responses, the effects were not long-lived and there were longer-term adverse sequelae $[3,4]$.

The next generation of PRRT agents developed was the more effective beta-emitting radionuclides conjugated to DOTA peptides. The first of these agents was ${ }^{90} \mathrm{Y}$ (labelled with DOTATOC and DOTATATE). Sustained symptomatic and objective responses with ${ }^{90} \mathrm{Y}$-PRRT were frequently observed $[5,6]$. However, an increased risk of significant permanent kidney damage with ${ }^{90} \mathrm{Y}$-PRRT has been reported. The Basel group reported that $9 \%$ of $>1,000$ patients treated with ${ }^{90} \mathrm{Y}$-DOTATOC developed chronic renal failure [7]. This renal toxicity is related to reabsorption of the radiopeptide in the proximal tubules, with the long path length of the ${ }^{90} \mathrm{Y}$ beta particle imparting a significant absorbed dose to the radiosensitive glomeruli.

${ }^{177} \mathrm{Lu}$ (labelled with DOTATATE or DOTATOC) was the next PRRT radiopharmaceutical to be developed. Over the last decade, ${ }^{177}$ Lu-DOTATATE (Lutathera ${ }^{\circledR}$ ) has become the most widely used PRRT radiopharmaceutical, with licensing by the European Marketing Authorisation granted in September 2017. ${ }^{177}$ Lu-DOTATATE was licensed following the positive results of the NETTER-1 trial, which showed a significantly prolonged progression-free survival of ${ }^{177} \mathrm{Lu}$-DOTATATE versus high-dose octreotide long-acting repeatable [8]. The de- gree of renal toxicity with ${ }^{177} \mathrm{Lu}$-DOTATATE is much less severe than that with ${ }^{90} \mathrm{Y}$-PRRT, with the Rotterdam group describing $<1 \%$ significant renal toxicity in over 500 patients treated [9]. Similarly, an analysis of $807 \mathrm{pa}$ tients treated with PRRT showed that treatment with ${ }^{90} \mathrm{Y}$ or ${ }^{90} \mathrm{Y}$ plus ${ }^{177} \mathrm{Lu}$ was more likely to cause nephrotoxicity compared with ${ }^{177} \mathrm{Lu}$ alone [10].

Despite the success of ${ }^{177} \mathrm{Lu}$-DOTATATE, patients invariably relapse, on average $2-3$ years after starting treatment. Many strategies are being explored to try to improve on and optimise the effectiveness of PRRT: some of these strategies include intra-arterial treatments (to better target liver metastases) [11], using dosimetry to ensure adequate tumour dosing [12], combining ${ }^{177} \mathrm{Lu}$-DOTATATE and ${ }^{90} \mathrm{Y}$ PRRT to target both small and large tumours [13], using chemotherapy as a PRRT radiosensitiser [14], a somatostatin receptor (SSR) antagonist PRRT (which has a higher SSR binding affinity) [15], and targeted alpha particle therapy (TAT) [16].

Treatment of cancer with TAT has been gaining popularity over the past few years, with TAT having been recently shown to be a successful treatment in prostate cancer in patients refractory to ${ }^{177} \mathrm{Lu}$-prostate-specific membrane antigen (PSMA) $[17,18]$. However, the evidence for TAT in NETs is sparse. In this review we discuss the physical differences between alpha and beta therapy as well as the potential advantages of alpha over beta particle therapy and review the currently available preclinical and clinical data for TAT in targeting the SSR in NETs. 
Table 1. Physical and biological differences between alpha and beta particles

\begin{tabular}{lll}
\hline & Alpha & Beta \\
\hline Particle type & ${ }^{4} \mathrm{He}$ nucleus & energetic electron \\
Particle energy & $5-9 \mathrm{MeV}$ & $50-2,300 \mathrm{keV}$ \\
Particle path length & $40-100 \mu \mathrm{m}$ & $0.05-12 \mathrm{~mm}$ \\
Linear energy transfer & $\sim 80 \mathrm{keV} / \mu \mathrm{m}$ & $\sim 0.2 \mathrm{keV} / \mu \mathrm{m}$ \\
Oxygenation & effective in hypoxic tumours & less effective in hypoxic tumours \\
Dose rates & linear exponential reduction in & high dose rates: tumour survival rates close \\
& tumour survival as absorbed dose & to linear exponential low dose rates: more \\
& increases & single-strand breaks (repairable) with \\
Bystander effect & shouldering of the dose-response curve \\
Tumour cross-fire & low & yes \\
\end{tabular}

\section{Physical Properties of Alpha and Beta Particles}

\section{Alpha Particles (Fig. 1)}

The physical characteristics of alpha and beta particles are shown in Table 1 [19]. Alpha particles are made up of two protons and two neutrons, with a structure similar to a ${ }^{4} \mathrm{He}$ nucleus without surrounding electrons (sometimes denoted as $\left.\mathrm{He}^{2+}\right)$. Alpha particles are produced in the process of alpha decay.

Alpha particles are positively charged and have a high particle energy ranging from 5 to $9 \mathrm{MeV}$ and a very short range of $40-100 \mu \mathrm{m}$. The range of the particle is thus equivalent to the thickness of 1-3 cell widths. Due to the short therapeutic range, intracellular accumulation of the alpha particle is preferred to ensure a higher chance of target damage to the cell's nucleus. Linear energy transfer (LET) is a term used in ionising radiation to measure the ionising density and hence molecular damage of a particle per unit length. LET is very high for alpha particles (80$100 \mathrm{keV} / \mu \mathrm{m})$ throughout its range and three times greater at the end of the path range (the Bragg peak) [20].

\section{Beta Particles (Fig. 1)}

Beta-emitting radioisotopes are negatively charged electrons that are emitted by a radioactive atom due to the process of beta decay. In beta decay, in an unstable nucleus a neutron is converted to a proton, and this process creates an energetic electron (beta particle). Beta particles are negatively charged, having a particle energy ranging from 50 to $2,300 \mathrm{keV}$, and have a path length from 0.0 to $12 \mathrm{~mm}$ [20]. The LET for beta decay is low $(0.2 \mathrm{keV} / \mu \mathrm{m})$ along the path length (i.e., they are sparsely ionising). Thus, many more particles are required for a similar absorbed dose compared with alpha particles.

\section{Radiobiology}

\section{Effects on DNA}

Ionising radiation deposits energy along a path at random. Energy deposition in the tumour cell nucleus will result in molecular changes in the target cell, which may result in cell death. Radiation damage to DNA triggers the cellular biological effects of radiation. There are a variety of different radiation effects on DNA, including singlestrand breaks, double-strand breaks, and multiply damaged DNA sites. Radiation that delivers double-strand breaks or multiple damaged sites is less likely to be repaired [20, 21].

In general, high-LET radiation results in more severe and less reparable cell damage than low-LET radiation [22-24]: alpha particles create a high ionisation density, which causes a high number of double-strand breaks as compared with beta particles which have a lower ionisation density (Fig. 2). The greater extent of double-strand breaks makes DNA repair more difficult and thus less effective. High-LET radiation also has indirect effects on tumours to make cell damage less likely to be repaired. These include an increased cell division time, being relatively independent of cell cycle phase, and reduced enzymatic repair mechanisms [25-27].

\section{Effect of Hypoxia}

Oxygen plays an important role in the biological effects of treatment, as plentiful oxygen results in a greater degree of free radical formation, which may block reparable chemical alterations [20]. Thus, hypoxia is more likely to attenuate the effects of ionising radiation, and the degree of tumour oxygenation can have a high impact on the radiosensitivity of tumours. The effect of hypoxia is 


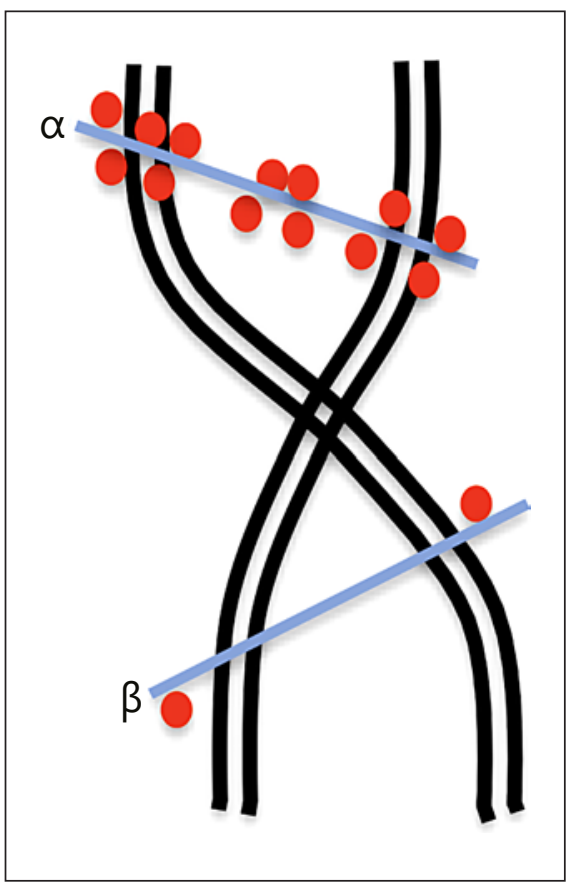

Fig. 2. Differences in LET between alpha and beta particles on DNA. Alpha particles have high LET $(\sim 80 \mathrm{keV} / \mu \mathrm{m})$ compared with the low LET $(\sim 0.2 \mathrm{keV} / \mu \mathrm{m})$ of beta particles. The high LET of alpha particles results in more double-strand breaks in DNA. LET, linear energy transfer.

high for low-LET radiation (i.e., beta emitters), but minimal for high-LET radiation. The reason for high-LET radiation being more resistant to hypoxic effects is that it produces reactive oxygen species which themselves result in cytotoxic effects in hypoxic cells [28].

\section{Characteristics of Therapeutic Alpha Radionuclides}

The physical properties of radionuclides used in alpha therapy are shown in Table 2. The first commercially available alpha therapy was ${ }^{223} \mathrm{Ra}\left(\right.$ Xofigo $\left.^{\circledR}\right)$, licensed for the treatment of bone metastases [29]; approximately $95 \%$ of emission energy is via alpha emission. As ${ }^{223} \mathrm{Ra}$ has a natural predilection for osteoblastic bone turnover, ${ }^{223} \mathrm{Ra}$ does not need to be labelled with an antibody/peptide to ensure targeting. Indeed, subsequent attempts to label ${ }^{223} \mathrm{Ra}$ with antibodies/peptides have not been successful, and thus ${ }^{223} \mathrm{Ra}$ has not been used in other TATs.

The two principal therapeutic radionuclides used in preclinical and clinical TAT of the SSR are ${ }^{213} \mathrm{Bi}$ and ${ }^{225} \mathrm{Ac}$. ${ }^{213} \mathrm{Bi}$ has a short half-life of $45 \mathrm{~min}$ and can be produced
Table 2. Physical properties of alpha-emitting particles used in alpha therapies

\begin{tabular}{llll}
\hline Radionuclide & $\begin{array}{l}\text { Mean energy, } \\
\mathrm{MeV}\end{array}$ & $\begin{array}{l}\text { Average path } \\
\text { length, } \mu \mathrm{m}\end{array}$ & Half-life \\
\hline${ }^{211} \mathrm{At}$ & 6.79 & 60 & $7.2 \mathrm{~h}$ \\
${ }^{213} \mathrm{Bi}$ & 8.32 & 84 & $45.6 \mathrm{~min}$ \\
${ }^{223} \mathrm{Ra}$ & 5.64 & 45 & 11.4 days \\
${ }^{225} \mathrm{Ac}$ & $6.83(27 \mathrm{MeV}$ & 61 & 10 days \\
& in total $)$ & & \\
\hline
\end{tabular}

from a ${ }^{225} \mathrm{Ac} /{ }^{213} \mathrm{Bi}$ generator, but the short half-life would require an on-site radiopharmacy to produce the TAT compound. This short half-life of ${ }^{213} \mathrm{Bi}$ could have some advantages: for an equivalent starting activity, higher dose rates given over a shorter period of time are more effective than low dose rates given over a longer period of time [30, 31]. Hofmann et al. [32] showed that $30 \mathrm{~min}$ following intravenous ${ }^{68} \mathrm{Ga}$-DOTATOC administration, $>80 \%$ of activity maximal accumulation in tumours was seen. Thus, it is likely a 45 -min half-life will be sufficient for ${ }^{213} \mathrm{Bi}$-PRRT to be incorporated into tumours via intravenous administration. ${ }^{213} \mathrm{Bi}$ has been successfully labelled with DOTA peptides with $>99 \%$ purity in preclinical and clinical studies $[16,27]$. The main mechanism of ${ }^{213} \mathrm{Bi}$ decay is via $435 \mathrm{keV}$ energy initial beta decay, followed almost instantly ( $3.7 \mu$ s delay) by alpha decay to ${ }^{209} \mathrm{~Pb}$ (3.2 h half-life) [20]. The alpha emission has a mean energy of $8.3 \mathrm{MeV} .{ }^{209} \mathrm{~Pb}$ subsequently decays to stable ${ }^{209} \mathrm{Bi}$. Posttherapeutic imaging of ${ }^{213} \mathrm{Bi}$ is possible, based on the $440 \mathrm{keV}$ gamma emission from the initial beta decay. However, the images are of poor quality due to bremsstrahlung production of ${ }^{209} \mathrm{~Pb}$, which results in a wide spectrum of lower-energy X-rays, impairing imaging quality.

${ }^{225} \mathrm{Ac}$ has a longer half-life of almost 10 days. ${ }^{225} \mathrm{Ac}$ is produced via decay of ${ }^{233} \mathrm{U}$ or from the neutron transmutation of ${ }^{226} \mathrm{Ra}$ to ${ }^{225} \mathrm{Ac}$ [21] at the US Department of Energy, Oak Ridge National Laboratory, and the Institute for Transuranium Elements in Karlsruhe, Germany. It has also been produced through a high-energy proton accelerator: ${ }^{227} \mathrm{Th}(\mathrm{p}, \mathrm{x})^{225} \mathrm{Ac}$ at Los Alamos National Laboratory [33]. However, production via radioactive decay and neutron irradiation methods lack the capacity to enable clinical use of ${ }^{225} \mathrm{Ac}$-radiolabelled peptides. Therefore, production via a high-energy proton accelerator at multiple sites will be required to enable the clinical use of ${ }^{225} \mathrm{Ac}$-radiolabelled drugs to treat NETs. ${ }^{225} \mathrm{Ac}$ has been 


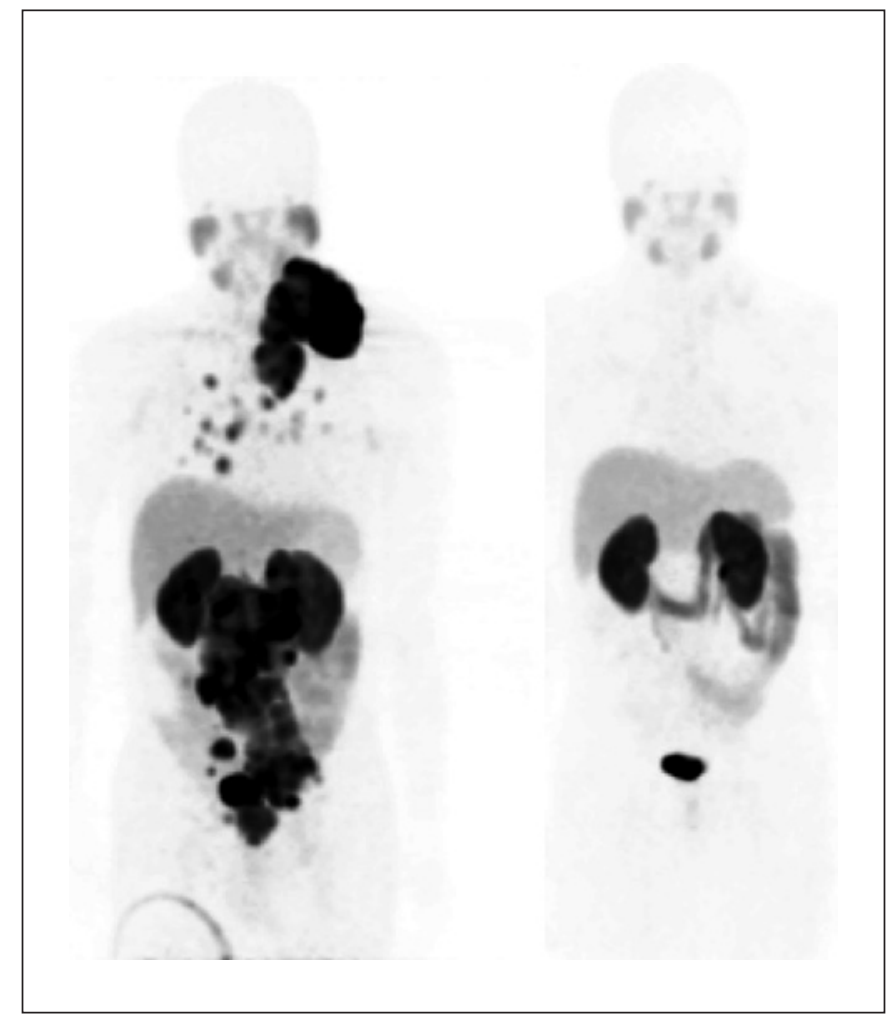

Fig. 3. ${ }^{68} \mathrm{Ga}-\mathrm{PSMA}$ studies in a patient with metastatic prostate cancer prior to (left) and after (right) ${ }^{225}$ Ac-PSMA TAT. There was an excellent functional response to treatment with concurrent clinical and biochemical response (prostate-specific antigen decreased from 1,301 to $0.05 \mathrm{ng} / \mathrm{L}$ ). Images courtesy of Prof. Mike Sathekge, Department of Nuclear Medicine, Steve Biko Academic Hospital, University of Pretoria, Pretoria, South Africa. PSMA, prostate-specific membrane antigen; TAT, targeted alpha particle therapy.

labelled with PSMA for the treatment of prostate cancer with a radiochemical purity of $>98 \%$ [17], although the radiochemical purity of ${ }^{225} \mathrm{Ac}$ labelled with DOTATOC in two preclinical studies has been slightly less (92 and $>95 \%)[34,35]$.

${ }^{225} \mathrm{Ac}$ has a decay scheme to stable ${ }^{209} \mathrm{Bi}$ (via a six-step process). During this decay, there is emission of four alpha particles (of 6-8 MeV energy each). The recoil effect after the alpha emission may result in the loss of the radiopeptide bond [16]. Thus, to maximise the effect and limit the toxicity of ${ }^{225} \mathrm{Ac}$, internalisation of the radiopeptide is important to ensure that all four alpha particles will act on the same target. The high overall alpha emission energies of $27.5 \mathrm{MeV}$ and half-life of 10 days renders ${ }^{225} \mathrm{Ac}$ a potentially appealing radionuclide for TAT. Post- therapeutic imaging of ${ }^{225} \mathrm{Ac}$ is possible, although images are also suboptimal (using gamma emissions of the daughter nuclides ${ }^{213} \mathrm{Bi}$ and ${ }^{221} \mathrm{Fr}$ ).

\section{TAT in Non-NET Cancers}

There have been several good recent reviews on alpha particle therapy in haematology and oncology [36-40]. These have described the use of TAT in several cancers including prostate, NET, breast, colon, myeloma, and ovarian cancer. Of these, prostate cancer is attracting the most interest. PSMA is the target overexpressed in prostate cancers. ${ }^{177} \mathrm{Lu}$-PSMA therapies have become an established treatment for patients with progressive metastatic prostate cancer. However, more recently there have been publications by German and South African groups describing the benefit of TAT. Kratochwil et al. [17] presented 2 patients ( 1 who had progressed through ${ }^{177} \mathrm{Lu}$ PSMA and 1 in whom ${ }^{177} \mathrm{Lu}$-PSMA was contraindicated due to diffuse bone marrow involvement) who went on to have TAT. Both these patients developed a complete clinical and biochemical response. Similarly, the South African group led by Sathekge et al. [18] demonstrated an excellent biochemical and molecular response in a patient treated with ${ }^{213} \mathrm{Bi}$-PSMA with large-volume disease. An image of a patient who had an excellent response to PSMA TAT is shown in Figure 3.

\section{Preclinical Studies Using TAT in SSR-Expressing \\ Tumours}

A summary of the preclinical studies performed in SSR-positive tumours is shown in Table 3. In an in vitro study by Nayak et al. [27] in human pancreatic adenocarcinoma cells, it was shown that the relative biological effectiveness of TAT using ${ }^{213} \mathrm{Bi}$-DOTATOC was 3.4 versus 1.0 for ${ }^{177} \mathrm{Lu}$-DOTATOC (i.e., at the same absorbed dose, ${ }^{213} \mathrm{Bi}$-DOTATOC has a 3.4 times greater therapeutic effect). Miederer et al. [34] investigated ${ }^{225}$ Ac-DOTATOC in mouse tumour xenograft implants; in their initial investigation they demonstrated that the highest non-toxic dose was $20 \mathrm{kBq} /$ mouse, while activities of $\geq 30 \mathrm{kBq}$ resulted in tubular necrosis. In the second part of their study, they evaluated the efficacy of ${ }^{225}$ Ac-DOTATOC (20 kBq/mouse) compared with ${ }^{177} \mathrm{Lu}$-DOTATOC (1 MBq/mouse) and with unlabelled DOTATOC. They found that 14 days after treatment the average tumour weight was significantly reduced in the ${ }^{225} \mathrm{Ac}$-DOTATOC arm (0.12 g) compared with ${ }^{177} \mathrm{Lu}$-DOTATOC (0.52 g) and controls $(0.89 \mathrm{~g})$. The mean energy of ${ }^{225} \mathrm{Ac}$ is 70 
Table 3. Summary of preclinical studies performed using TAT

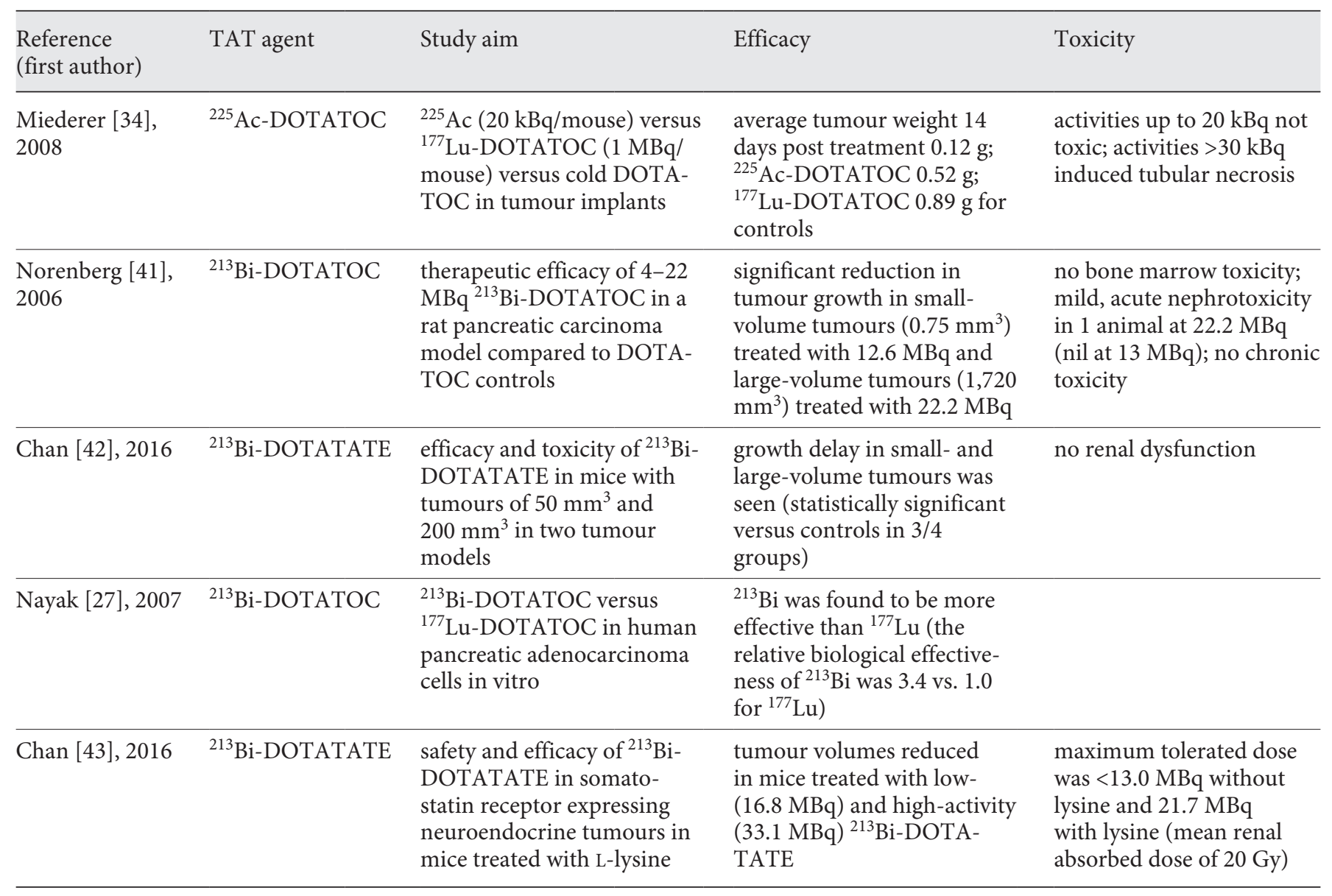

TAT, targeted alpha particle therapy.

times greater than that of ${ }^{177} \mathrm{Lu}(27$ vs. $0.4 \mathrm{MeV})$. The therapeutic activity ratio of ${ }^{225} \mathrm{Ac}$ versus ${ }^{177} \mathrm{Lu}$ in that study was approximately 50 , which is in favour of ${ }^{225} \mathrm{Ac}$. However, the authors pointed out that this ratio difference was offset by the lower radiochemical purity of ${ }^{225}$ Ac-DOTATOC versus ${ }^{177}$ Lu-DOTATOC (92 vs. 99\%). Thus, the observed difference in tumour weights in the two groups is unlikely to have been caused by the activity ratio difference, but more likely to be related to the relative biological effectiveness of TAT.

Norenberg et al. [41] investigated ${ }^{213} \mathrm{Bi}$-DOTATOC in a rat pancreatic carcinoma model (compared to DOTATOC controls). They were successful in labelling this compound with high radiochemical purity, and it was stable in serum for $>24 \mathrm{~h}$. There was a significant reduction in tumour growth in rats with both small tumours $\left(0.75 \mathrm{~mm}^{3}\right.$, treated with $\left.12.6 \mathrm{MBq}\right)$ and large tumours $\left(1,720 \mathrm{~mm}^{3}\right.$, treated with $\left.22.2 \mathrm{MBq}\right)$ compared with
DOTATOC controls. There was 1 case of acute mild nephrotoxicity at $22.2 \mathrm{MBq}$, but no chronic nephrotoxicity was seen. There were no acute or chronic haematological toxicities. Of note, that study involved a syngeneic tumour - a rat tumour in an immune-competent rat host. The efficacy of TAT in syngeneic tumours is more significant as compared to xenografts to immunocompromised animals.

In another ${ }^{213} \mathrm{Bi}$-PRRT study, Chan et al. [42] used ${ }^{213} \mathrm{Bi}$-DOTATATE in mice bearing tumours of different sizes (50 and $200 \mathrm{~mm}^{3}$ ) using two tumour models (H69 human small-cell lung carcinoma and CA20948 rat pancreatic tumour). A delay in tumour growth and higher survival was seen in both models and both tumour sizes. In 3/18 animals with a small tumour type, there was no tumour regrowth at 90 days. No renal dysfunction occurred at activities of $2-4 \mathrm{MBq}$. This group also demonstrated that lysine reduced the renal dose by $50 \%$, with a 
maximal tolerated renal dose of $13 \mathrm{MBq}$ without lysine and of $21 \mathrm{MBq}$ with lysine renoprotection [43].

\section{Clinical Studies Using TAT in SSR-Expressing}

\section{Tumours}

The efficacy of TAT in preclinical studies prompted the Heidelberg group to investigate TAT clinically. The first-in-human TAT study in NETs was published in 2014: Kratochwil et al. [16] presented a group of 8 patients with progressive NETs who were refractory to ${ }^{90} \mathrm{Y} /{ }^{177} \mathrm{Lu}$-DOTATOC treatment; $7 / 8$ patients were treated with the intra-arterial administration of ${ }^{213} \mathrm{Bi}$-DOTATOC (usually via the hepatic artery) while $1 / 8$ patients had an intravenous administration. Of the 7 intra-arterial administrations, 1 patient developed a complete response (to liver and pancreatic primary tracer delivered via gastroduodenal artery to target both primary and metastases), 2 patients had a sustained partial response, 3 patients had stable disease, and 1 patient developed a radiological response to the liver tumours (skeletal metastases not targeted/assessed). Interestingly, 1 patient was felt unsuitable for beta-emitting PRRT due to diffuse liver involvement, with little healthy liver (high risk for hepatic decompensation). This patient had a substantial response to TAT in the liver, with the liver enzymes having returned to normal. Intriguingly, in some patients there was targeting of tumours outside the liver (bone metastases) despite the intra-arterial route of administration. This was probably related to tracer redistribution following the first-pass effect. The last patient had diffusely disseminated bone marrow metastases with beta-emitting PRRT thus relatively contraindicated. This patient had one cycle of systemic TAT: the bone marrow tumours were well targeted with no significant bone marrow toxicity (response assessment was not possible on cross-sectional imaging).

In the evaluation of toxicity, there was a mean reduction in glomerular filtration rate of $30 \%$ and a tubular extraction rate of $40 \% 2$ years following TAT. The mean glomerular filtration rate was $115 \mathrm{~mL} / \mathrm{min}$ at baseline and $83 \mathrm{~mL} / \mathrm{min}$ at 2 years. One patient had developed a creatinine value of $1-1.5 \times$ the upper limit of normal 2 years after treatment. It should be noted, however, that all these patients had excellent baseline renal function, and thus it was not possible to assess the effects in patients with a lower glomerular filtration rate or mildly impaired renal function.

There was a low amount of acute haematological toxicity. One patient with previous grade IV thrombocytopenia following ${ }^{90} \mathrm{Y}$-DOTATOC therapy developed grade 2 thrombocytopenia following ${ }^{213} \mathrm{Bi}$-DOTATOC. Other patients developed no toxicity or grade 1 haematological toxicity. On follow-up, 3 patients developed chronic anaemia, one of whom was diagnosed with myelodysplastic syndrome followed by acute myeloid leukaemia.

At present there are no other published clinical studies for TAT in NETs. Radiomedix is sponsoring a US-based trial of TAT in NETs using ${ }^{212} \mathrm{~Pb}$-DOTAMTATE (AlphaMedix $^{\mathrm{TM}}$ ). This is a phase 1 dose escalation study in PRRTnaïve patients [44], the results of which are awaited with keen interest.

\section{Conclusions}

Clinicians have not until very recently been much aware of the therapeutic benefits of alpha particle radiation. Clinically, targeted alpha SSR therapy is an exciting and promising therapeutic modality for SSR-expressing NETs. TAT has potential advantages over beta-emitting therapy due to the high energy and short path length of alpha particles causing double-stranded breaks in DNA and the relative tolerance of alpha particles to cell cycle effects and hypoxic conditions, thus getting "more bangs for your buck." Preclinical studies have shown that it may be more efficacious than beta-emitting particles in PRRTnaïve animals, with limited short-term bone marrow toxicity, although TAT-related renal dysfunction is at present largely unknown. Preclinical and early clinical studies have shown that TAT provides tumoural responses in patients who have become resistant or are unsuitable for beta-emitting PRRT. Due to the high LET of TAT and associated renal tubular damage, the kidney may prove to be the critical organ for TAT. The initial focus of clinical studies should be on phase 1 studies to establish tolerated renal dosing. Ideally, a partnership with a pharmaceutical company to fast-track TAT will help bring this promising treatment quickest to the global market.

\section{Disclosure Statement}

The authors declare no conflict of interest. 


\section{References}

1 Ramage JK, Ahmed A, Ardill J, Bax N, Breen DJ, Caplin ME, et al.; UK and Ireland Neuroendocrine Tumour Society. Guidelines for the management of gastroenteropancreatic neuroendocrine (including carcinoid) tumours (NETs). Gut. 2012 Jan;61(1):6-32.

2 Rindi G, Klöppel G, Couvelard A, Komminoth P, Körner M, Lopes JM, et al. TNM staging of midgut and hindgut (neuro) endocrine tumors: a consensus proposal including a grading system. Virchows Arch. 2007 Oct; 451(4):757-62.

3 Valkema R, de Jong M, Bakker WH, Breeman WA, Kooij PP, Lugtenburg PJ, et al. Phase I study of peptide receptor radionuclide therapy with [In-DTPA] octreotide: the Rotterdam experience. Semin Nucl Med. 2002 Apr;32(2): 110-22.

4 Buscombe JR, Caplin ME, Hilson AJ. Longterm efficacy of high-activity 111 in-pentetreotide therapy in patients with disseminated neuroendocrine tumors. J Nucl Med. 2003 Jan;44(1):1-6.

5 Bushnell DL Jr, O’Dorisio TM, O’Dorisio MS, Menda Y, Hicks RJ, Van Cutsem E, et al. 90Yedotreotide for metastatic carcinoid refractory to octreotide. J Clin Oncol. 2010 Apr; 28(10):1652-9.

6 Cwikla JB, Sankowski A, Seklecka N, Buscombe JR, Nasierowska-Guttmejer A, Jeziorski KG, et al. Efficacy of radionuclide treatment DOTATATE Y-90 in patients with progressive metastatic gastroenteropancreatic neuroendocrine carcinomas (GEP-NETs): a phase II study. Ann Oncol. 2010 Apr;21(4): 787-94.

7 Imhof A, Brunner P, Marincek N, Briel M, Schindler C, Rasch H, et al. Response, survival, and long-term toxicity after therapy with the radiolabeled somatostatin analogue [90YDOTA]-TOC in metastasized neuroendocrine cancers. J Clin Oncol. 2011 Jun;29(17): 2416-23.

8 Strosberg J, El-Haddad G, Wolin E, Hendifar A, Yao J, Chasen B, et al.; NETTER-1 Trial Investigators. Phase 3 trial of $177 \mathrm{Lu}$-dotatate for midgut neuroendocrine tumors. $\mathrm{N}$ Engl J Med. 2017 Jan;376(2):125-35.

9 Kwekkeboom DJ, de Herder WW, Kam BL, van Eijck CH, van Essen M, Kooij PP, et al. Treatment with the radiolabeled somatostatin analog [177 Lu-DOTA 0,Tyr3]octreotate: toxicity, efficacy, and survival. J Clin Oncol. 2008 May;26(13):2124-30.

10 Bodei L, Kidd M, Paganelli G, Grana CM, Drozdov I, Cremonesi M, et al. Long-term tolerability of PRRT in 807 patients with neuroendocrine tumours: the value and limitations of clinical factors. Eur J Nucl Med Mol Imaging. 2015 Jan;42(1):5-19.
11 Kratochwil C, López-Benítez R, Mier W, Haufe S, Isermann B, Kauczor HU, et al. Hepatic arterial infusion enhances DOTATOC radiopeptide therapy in patients with neuroendocrine liver metastases. Endocr Relat Cancer. 2011 Sep;18(5):595-602.

12 Bison SM, Konijnenberg MW, Melis M, Pool SE, Bernsen MR, Teunissen JJ, et al. Peptide receptor radionuclide therapy using radiolabeled somatostatin analogs: focus on future developments. Clin Transl Imaging. 2014; 2(1):55-66

13 Villard L, Romer A, Marincek N, Brunner P, Koller MT, Schindler C, et al. Cohort study of somatostatin-based radiopeptide therapy with [(90)Y-DOTA]-TOC versus [(90)YDOTA]-TOC plus [(177)Lu-DOTA]-TOC in neuroendocrine cancers. J Clin Oncol. 2012 Apr;30(10):1100-6.

14 Claringbold PG, Price RA, Turner JH. Phase I-II study of radiopeptide $177 \mathrm{Lu}$-octreotate in combination with capecitabine and temozolomide in advanced low-grade neuroendocrine tumors. Cancer Biother Radiopharm. 2012 Nov;27(9):561-9.

15 Wild D, Fani M, Fischer R, Del Pozzo L, Kaul F, Krebs S, et al. Comparison of somatostatin receptor agonist and antagonist for peptide receptor radionuclide therapy: a pilot study. J Nucl Med. 2014 Aug;55(8):1248-52.

16 Kratochwil C, Giesel FL, Bruchertseifer F, Mier W, Apostolidis C, Boll R, et al. (213)BiDOTATOC receptor-targeted alpha-radionuclide therapy induces remission in neuroendocrine tumours refractory to beta radiation: a first-in-human experience. Eur J Nucl Med Mol Imaging. 2014 Nov;41(11):2106-19.

17 Kratochwil C, Bruchertseifer F, Giesel FL, Weis M, Verburg FA, Mottaghy F, et al. 225Ac-PSMA-617 for PSMA-targeted $\alpha$-radiation therapy of metastatic castration-resistant prostate cancer. J Nucl Med. 2016 Dec; 57(12):1941-4.

18 Sathekge M, Knoesen O, Meckel M, Modiselle M, Vorster M, Marx S. 213Bi-PSMA-617 targeted alpha-radionuclide therapy in metastatic castration-resistant prostate cancer. Eur J Nucl Med Mol Imaging. 2017 Jun;44(6): 1099-100.

19 Huang CY, Guatelli S, Oborn BM, Allen BJ. Microdosimetry for targeted alpha therapy of cancer. Comput Math Methods Med. 2012; 2012:153212.

20 Kassis AI. Therapeutic radionuclides: biophysical and radiobiologic principles. Semin Nucl Med. 2008 Sep;38(5):358-66.

21 Scheinberg DA, McDevitt MR. Actinium-225 in targeted alpha-particle therapeutic applications. Curr Radiopharm. 2011 Oct;4(4):30620.

22 Goodhead DT, Thacker J, Cox R. Weiss Lecture. Effects of radiations of different qualities on cells: molecular mechanisms of damage and repair. Int J Radiat Biol. 1993 May;63(5): 543-56.
23 Goodhead DT. Mechanisms for the biological effectiveness of high-LET radiations. J Radiat Res (Tokyo). 1999 Dec;40 Suppl:1-13.

24 Friesen C, Glatting G, Koop B, Schwarz K, Morgenstern A, Apostolidis C, et al. Breaking chemoresistance and radioresistance with [213Bi]anti-CD45 antibodies in leukemia cells. Cancer Res. 2007 Mar;67(5):1950-8.

25 Tobias CA, Blakely EA, Alpen EL, Castro JR, Ainsworth EJ, Curtis SB, et al. Molecular and cellular radiobiology of heavy ions. Int J Radiat Oncol Biol Phys. 1982 Dec;8(12):210920.

26 Dodson H, Wheatley SP, Morrison CG. Involvement of centrosome amplification in radiation-induced mitotic catastrophe. Cell Cycle. 2007 Feb;6(3):364-70.

27 Nayak TK, Norenberg JP, Anderson TL, Prossnitz ER, Stabin MG, Atcher RW. Somatostatin-receptor-targeted alpha-emitting $213 \mathrm{Bi}$ is therapeutically more effective than beta(-)-emitting 177Lu in human pancreatic adenocarcinoma cells. Nucl Med Biol. 2007 Feb;34(2):185-93.

28 Narayanan PK, Goodwin EH, Lehnert BE. Alpha particles initiate biological production of superoxide anions and hydrogen peroxide in human cells. Cancer Res. 1997 Sep;57(18): 3963-71.

29 Parker C, Nilsson S, Heinrich D, Helle SI, O’Sullivan JM, Fosså SD, et al.; ALSYMPCA Investigators. Alpha emitter radium-223 and survival in metastatic prostate cancer. $\mathrm{N}$ Engl J Med. 2013 Jul;369(3):213-23.

30 O'Donoghue JA. The impact of tumor cell proliferation in radioimmunotherapy. Cancer. 1994 Feb;73(3 Suppl):974-80.

31 Barendsen GW. LET dependence of linear and quadratic terms in dose-response relationships for cellular damage: correlations with the dimensions and structures of biological targets. Radiat Prot Dosimetry. 1990; 31(1-4):235-9.

32 Hofmann M, Maecke H, Börner R, Weckesser E, Schöffski P, Oei L, et al. Biokinetics and imaging with the somatostatin receptor PET radioligand (68)Ga-DOTATOC: preliminary data. Eur J Nucl Med. 2001 Dec;28(12):17517.

33 Griswold JR, Medvedev DG, Engle JW, Copping R, Fitzsimmons JM, Radchenko V, et al. Large scale accelerator production of $225 \mathrm{Ac}$ : effective cross sections for $78-192 \mathrm{MeV}$ protons incident on 232Th targets. Appl Radiat Isot. 2016 Dec;118:366-74.

34 Miederer M, Henriksen G, Alke A, Mossbrugger I, Quintanilla-Martinez L, SenekowitschSchmidtke R, et al. Preclinical evaluation of the alpha-particle generator nuclide 225Ac for somatostatin receptor radiotherapy of neuroendocrine tumors. Clin Cancer Res. 2008 Jun;14(11):3555-61.
Alpha Particle Therapy for

Neuroendocrine Tumours
Neuroendocrinology 2019;108:256-264 DOI: $10.1159 / 000494760$ 
35 Graf F, Fahrer J, Maus S, Morgenstern A, Bruchertseifer F, Venkatachalam S, et al. DNA double strand breaks as predictor of efficacy of the alpha-particle emitter Ac-225 and the electron emitter Lu-177 for somatostatin receptor targeted radiotherapy. PLoS One. 2014 Feb;9(2):e88239.

36 Marcu L, Bezak E, Allen BJ. Global comparison of targeted alpha vs targeted beta therapy for cancer: in vitro, in vivo and clinical trials. Crit Rev Oncol Hematol. 2018 Mar; 123:7-20.

37 Makvandi M, Dupis E, Engle JW, Nortier FM, Fassbender ME, Simon S, et al. Alpha-Emitters and Targeted Alpha Therapy in Oncology: from Basic Science to Clinical Investigations. Target Oncol. 2018 Apr;13(2):189-203.
38 Poty S, Francesconi LC, McDevitt MR, Morris MJ, Lewis JS. a-Emitters for radiotherapy: from basic radiochemistry to clinical studies part 1. J Nucl Med. 2018 Jun;59(6):878-84.

39 Poty S, Francesconi LC, McDevitt MR, Morris MJ, Lewis JS. a-Emitters for radiotherapy: from basic radiochemistry to clinical studies part 2. J Nucl Med. 2018 Jul;59(7):1020-7.

40 Morgenstern A, Apostolidis C, Kratochwil C, Sathekge M, Krolicki L, Bruchertseifer F. An overview of targeted alpha therapy with 225Actinium and 213Bismuth. Curr Radiopharm. 2018 May;11(3):200-8.

41 Norenberg JP, Krenning BJ, Konings IR, Kusewitt DF, Nayak TK, Anderson TL, et al. 213Bi-[DOTA0, Tyr3] octreotide peptide receptor radionuclide therapy of pancreatic tumors in a preclinical animal model. Clin Cancer Res. 2006 Feb;12(3 Pt 1):897-903.
42 Chan HS, Konijnenberg MW, de Blois E, Koelewijn S, Baum RP, Morgenstern A, et al. Influence of tumour size on the efficacy of targeted alpha therapy with (213) Bi[DOTA(0),Tyr(3)]-octreotate. EJNMMI Res. 2016 Dec;6(1):6.

43 Chan HS, Konijnenberg MW, Daniels T, Nysus $\mathrm{M}$, Makvandi M, de Blois E, et al. Improved safety and efficacy of 213Bi-DOTATATE-targeted alpha therapy of somatostatin receptor-expressing neuroendocrine tumors in mice pre-treated with L-lysine. EJNMMI Res. 2016 Dec;6(1):83.

44 Phase 1 study of AlphaMedix(TM) in adult subjects with SSTR (+) NET. https://clinicaltrials.gov/ct2/show/NCT03466216. 